\title{
Simultaneous broadband generation of second and third harmonics from chirped nonlinear photonic crystals
}

\author{
Bao-Qin Chen ${ }^{1}$, Ming-Liang Ren ${ }^{1}$, Rong-Juan Liu ${ }^{1}$, Chao Zhang ${ }^{1}$, Yan Sheng ${ }^{2}$, Bo-Qin Ma ${ }^{3}$ and Zhi-Yuan Li ${ }^{1}$ \\ Ultrabroadband laser sources are highly desirable in a wide variety of modern science disciplines ranging from physics, chemistry and \\ materials science to information communications and processing. Here we present the design and fabrication of a chirped periodically \\ poled lithium niobate (CPPLN) nonlinear photonic crystal that supports multiple orders of quasiphase matching with finite bandwidth \\ and allows for the simultaneous broadband generation of second and third harmonics with high conversion efficiency. Moreover, the \\ chirp rate has a significant influence on the conversion efficiency and bandwidth. The CPPLN scheme offers a promising approach for \\ the construction of short-wavelength laser sources and enables the generation of the three primary colors—red, green and blue-from a \\ single crystal, which may have potential applications in large-screen laser displays.
}

Light: Science \& Applications (2014) 3, e189; doi:10.1038/lsa.2014.70; published online 18 July 2014

Keywords: chirped periodically poled lithium niobate; quasiphase matching; second-harmonic generation; third-harmonic generation

\section{INTRODUCTION}

Ultrabroadband laser sources are highly desirable in a wide variety of modern science disciplines ranging from physics, chemistry and materials science to information communications and processing. Laser gain media that are capable of short-pulse generation are available but limited, and they cover only a small portion of the optical spectrum. Shortly after the creation of the first laser in 1960, the door to nonlinear optics was opened because of the discovery of second-harmonic generation (SHG). ${ }^{1}$ Broad-bandwidth and high-conversion-efficiency SHG, third-harmonic generation (THG), higher-order-harmonic generation and various frequency-mixing and parametric-conversion processes have all provided fascinating routes toward the considerable expansion of the spectral range of laser sources.

To realize high-efficiency SHG, THG and other optical parametric processes, nonlinear optical materials are required to simultaneously satisfy the phase-matching condition and possess large nonlinear optical coefficients in the phase-matching direction. Although many natural nonlinear crystals have large nonlinear coefficients in certain directions, proper phase matching cannot always be achieved because of the dispersion of the material. Thus, the optical birefringent properties of certain birefringent nonlinear crystals are often utilized to compensate for the material dispersion and achieve phase matching. However, the stringent conditions for birefringent phase matching greatly limit the extension of the optical frequency. As an alternative approach, quasiphase matching (QPM), which was first proposed in $1962,{ }^{2}$ launched a new era in nonlinear optics because of several advantages it offers over conventional birefringence phase matching in the frequency-conversion process. These advantages include phase matching in materials that have high nonlinear optical coefficients but low or no birefringence, the utilization of the highest element of the nonlinear susceptibility tensor, the suppression of the walk-off effect and considerable flexibility in the choice of wavelengths involved in nonlinear optical frequency mixing. ${ }^{3-6}$ QPM has been successfully demonstrated in many one-dimensional and two-dimensional nonlinear periodic, quasiperiodic and aperiodic lattices as well as superlattices $^{7-9}$ to achieve high-efficiency SHG. The QPM scheme is equally effective for THG. Cascaded processes involving successive SHG and sum-frequency generation (SFG) in a single QPM crystal can be used to create THG. ${ }^{10-14}$

In practice, in addition to a broad bandwidth, high conversion efficiency is also desired. However, there is a tradeoff between conversion efficiency and bandwidth. For example, an effective apodization technique has been proposed to broaden and flatten the wavelengthconversion bandwidth by engineering patterns of QPM step-chirped gratings in poled lithium niobate $\left(\mathrm{LiNbO}_{3}\right)$ waveguides. ${ }^{9}$ This technique has been used to achieve bandwidths of approximately $40 \mathrm{~nm}$ with SHG conversion efficiencies of approximately -12 to $-20 \mathrm{~dB}$ $(6.3 \%-1 \%)$. In the case of another design based on engineered inverted domains in the form of step-chirped gratings in $\mathrm{MgO}$-doped poled $\mathrm{LiNbO}_{3}$ waveguides, the achieved efficiency curves have indicated a bandwidth as large as $65 \mathrm{~nm}$ and an SHG conversion efficiency of $-22.5 \mathrm{~dB}(\sim 0.56 \%) .{ }^{15} \mathrm{~A} \mathrm{QPM} \mathrm{LiTaO}_{3}$ waveguide has been proposed and demonstrated to obtain a bandwidth of $0.35 \mathrm{~nm}$ with an efficiency of $57 \%$ through modulation of the waveguide width and a bandwidth of $1.12 \mathrm{~nm}$ with an efficiency of $29 \%$ through modulation of the grating period. ${ }^{16}$

Previous studies have demonstrated that the origin of this tradeoff lies in group-velocity mismatch and group-velocity dispersion. ${ }^{17}$

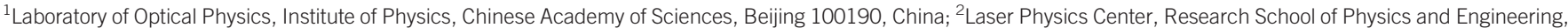
Australian National University, Canberra, ACT 0200, Australia and ${ }^{3}$ College of Sciences, Communication University of China, Beijing 100024 , China 
These effects lead to the decrease of the conversion efficiency and the increase of the pulse width in the frequency-conversion process. The use of shorter crystals is an effective method of solving this problem, but when the nonlinear interaction distance is shorter, the conversion efficiency also decreases. Therefore, aperiodic QPM structures have been proposed to circumvent this tradeoff. Aperiodic structures have a broader spectral response than periodic ones. ${ }^{3}$ For example, a QPM structure with a linearly varying wave vector along the direction of wave propagation can be used to achieve effective nonlinear pulse compression $^{18,19}$ and to implement various frequency-conversion processes. $^{20-23}$

In this work, we achieve a considerable advancement in this technology through the design and fabrication of chirped periodically poled lithium niobate (CPPLN) nonlinear crystals whose modulation period of nonlinear susceptibility varies along the propagation direction. We demonstrate both theoretically and experimentally that CPPLN can support multiple orders of QPM with finite bandwidth and therefore, allows for simultaneous broadband SHG and THG with high conversion efficiency from a single crystal.

\section{MATERIALS AND METHODS}

\section{Principle of the CPPLN scheme}

The CPPLN nonlinear crystal has a modulation period of nonlinear susceptibility that varies along the propagation direction. This feature can produce continuously varied reciprocal lattice vectors (RLVs) to compensate the phase mismatching at various wavelengths through the mechanism of chirped QPM. The poled period of the CPPLN crystal is given by $\Lambda(z)=\Lambda_{0} /\left[1+\left(D_{g} \Lambda_{0} z / 2 \pi\right)\right]$ along the light-propagation direction (denoted as $+z$ ). Here $\Lambda_{0}=2 \pi / \Delta k_{0}$, and $D_{g}$ is the chirp rate. The wave-vector mismatch $\Delta k_{0}=4 \pi\left[n_{2}\left(\lambda_{0}\right)-n_{1}\left(\lambda_{0}\right)\right] / \lambda_{0}$ is defined for SHG at a selected value of the fundamental wavelength, which is $\lambda_{0}=1.4 \mu \mathrm{m}$. The central period of this CPPLN structure is deliberately designed to achieve first-order QPM for SHG at $1.4 \mu \mathrm{m}$. As illustrated in Figure 1a, the width of the negative domains in the CPPLN structure, $l_{-}$, is fixed to a certain value, and a chirped poling period is obtained by varying the widths of the positive domains, $l_{+}$. The width of the negative domain is fixed to $l_{-}=6 \mu \mathrm{m}$. To investigate the effect of the chirp rate on the bandwidth, we designed three chirped structures with different chirp rates: $D_{g 1}=0.6, D_{g 2}=1.2$ and $D_{g 3}=2.4$. The poling period is $\Lambda(z)=l_{+}(z)+l_{-}$. The sample length is approximately $L=16 \mathrm{~mm}$. The width of the positive domain is gradually reduced along the propagation direction, and the corresponding period reduces from 15.276 to $14.595 \mu \mathrm{m}$ for $D_{g 1}=0.6$, from 15.641 to $14.277 \mu \mathrm{m}$ for $D_{g 2}=1.2$ and from 16.426 to $13.681 \mu \mathrm{m}$ for $D_{g 3}=2.4$ along the light-propagation direction. The average period is $14.928 \mu \mathrm{m}$, which satisfies the first-order QPM requirement for SHG at $1.4 \mu \mathrm{m}$.

For a traditional PPLN structure, the effective wave-vector mismatch is given by $\Delta k(\lambda)=4 \pi\left[n_{2}(\lambda)-n_{1}(\lambda)\right] / \lambda-G_{m}$, where $G_{m}=2 m \pi / \Lambda . \Lambda$ is the poling period of the PPLN structure and $m$ represents the QPM order. The poling periods requiring for the first-order QPM of SHG at $1.1,0.966$ and $0.888 \mu \mathrm{m}$ are $7.464,4.976$ and $3.732 \mu \mathrm{m}$, respectively. The average period of our CPPLN structure is $14.928 \mu \mathrm{m}$, which can achieve SHG at wavelengths of 1.1, 0.966 and $0.888 \mu \mathrm{m}$, corresponding to second-, third- and fourth-order QPM, respectively. The local effective wave-vector mismatch $\Delta k$ for this CPPLN structure is defined as $\Delta k(\lambda, z)=4 \pi\left[n_{2}(\lambda)-n_{1}(\lambda)\right] / \lambda-G_{m}(z)$, where $G_{m}(z)=2 m \pi / \Lambda(z)$. As the poling period $\Lambda(z)$ decreases along the propagation direction, the reciprocal vector $G_{m}(z)$ gradually increases. Therefore, the local effective wave-vector mismatch $\Delta k(\lambda, z)$ of our CPPLN structure can
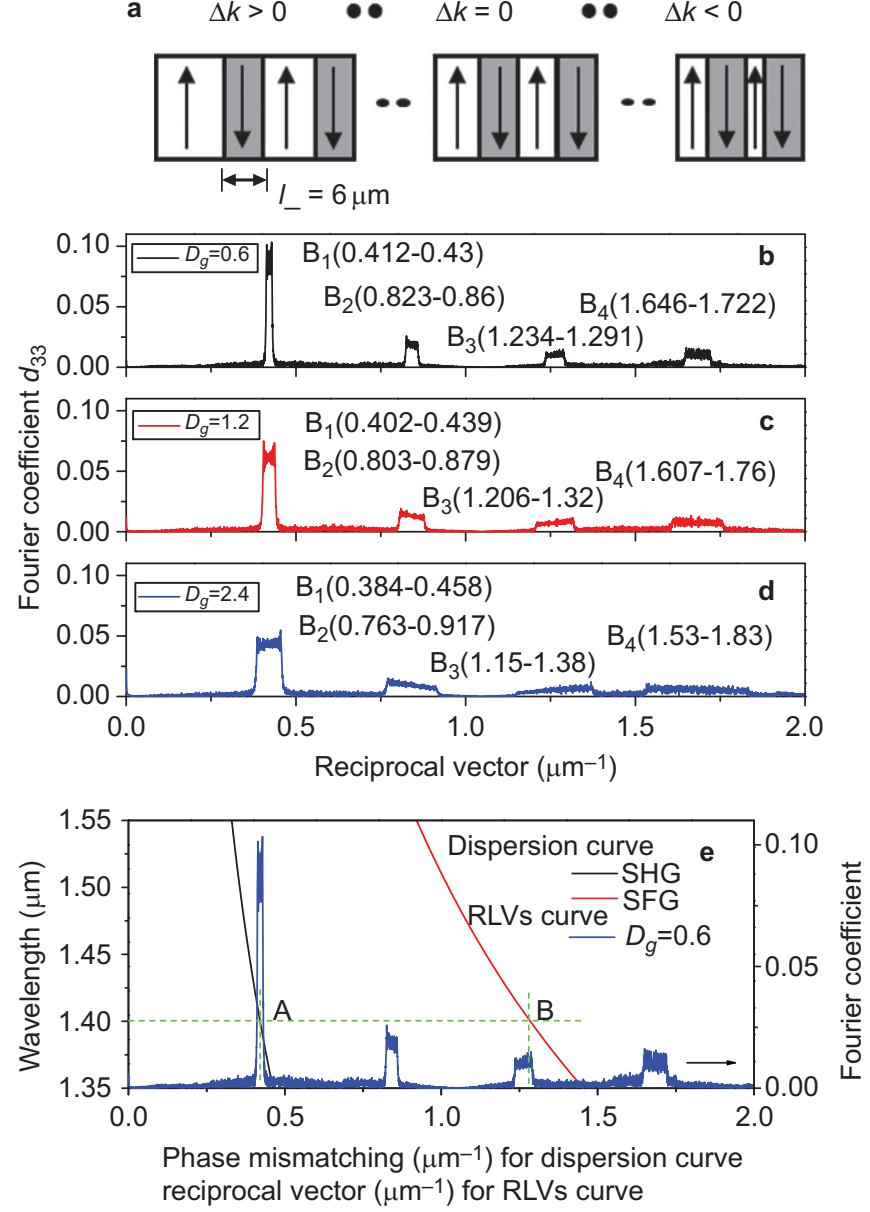

Figure 1 Principle of the CQPM scheme in CPPLN for broadband SHG and THG. (a) Schematic diagrams of the structural geometry of a CPPLN structure and the corresponding phase-mismatching distribution properties. The up or down arrows indicate positive or negative domains, respectively. The width of each negative domain is fixed to $I_{-}=6 \mu \mathrm{m}$. (b-d) Calculated distributions of the nonlinear coefficient as functions of the RLVs for the three investigated chirp rates. Four continuous RLV bands, which correspond to first-, second-, third- and fourth-order QPM, are apparent. The nonlinear coefficients are expressed in units of the second-order nonlinear coefficient $d_{33}$ of $\mathrm{LiNbO}_{3}$, which is equal to $27.2 \mathrm{pm} \mathrm{V}$. These bands can be used for broadband and multiwavelength frequency conversion. (e) Combined plots of the phase-mismatch curves for SHG and SFG in a $\mathrm{LiNbO}_{3}$ crystal and the nonlinear-coefficient curve for the CPPLN structure with a chirp rate of $D_{g}=0.6$. This plot illustrates the mechanisms of SHG, SFG and THG in these CPPLN structures. Points A and B denote the wavelength band (approximately $1.40 \mu \mathrm{m})$ in which SHG (FW+FW) and SFG (FW+SHW) can occur simultaneously because of the fulfillment of the QPM condition, leading to simultaneous broadband SHG and THG from this CPPLN structure. CPPLN, chirped periodically poled lithium niobate; CQPM, chirped quasiphase matching; FW, fundamental wave; QPM, quasiphase matching; RLV, reciprocal lattice vector; SFG, sum-frequency generation; SHG, second-harmonic generation; SHW, second-harmonic wave; THG, third-harmonic generation.

continuously vary at a given wavelength $\lambda$. For a wavelength near $\lambda_{0}$, there will always be a certain value of $G_{m}(z)=\Delta k(\lambda)$ that allows for completely compensation of the phase mismatch. Thus, our CPPLN structure will always have an effective wave-vector mismatch that varies from $\Delta k>0$ to $\Delta k<0$, as demonstrated in Figure 1a.

The RLV and the period constant are reciprocal to each other. Numerically, the RLV composition of the structure can be calculated by applying a Fourier transform to the domain-structure position 
function, which takes a value of +1 for positive domains and a value of -1 for negative domains. Figure $1 b-1 d$ illustrates that the CPPLN structures have four continuous bands of RLVs with high effective nonlinear susceptibility, ${ }^{24}$ and the bandwidth increases as the chirp rate increases. This phenomenon is simple to understand. A CPPLN structure with a larger chirp rate has a larger range of variation in the size of its period. According to Fourier analysis, the variation range of the RLV around each order (e.g., first-, second-, third- and fourthorder) of QPM for a perfect PPLN is therefore also larger.

These continuous bands of RLVs enable broadband and multiwavelength frequency conversion, not only for SHG but also for SFG and THG. To clearly illustrate this fact, in Figure 1e, the RLVs curve for the CPPLN structure with $D_{g 1}=0.6$ is plotted alongside the phase-mismatch curves for both SHG and SFG. The SHG and SFG curves both intersect with the RLVs curve several times throughout a very broad wavelength range from the infrared to the visible region, and each has a finite bandwidth. This means that the CPPLN structure enables multicolor broadband SHG and SFG. In addition, the SHG and SFG cross points (points A and B) occur in the same wavelength band at approximately $1.40 \mu \mathrm{m}$. This means that simultaneous broadband SHG and THG can occur in this CPPLN structure at approximately $1.40 \mu \mathrm{m}$.

\section{Fabrication of CPPLN samples}

We fabricated the CPPLN samples using the electric poling technique at room temperature. Standard optical-grade $\mathrm{Z}$-cut $\mathrm{LiNbO}_{3}$ crystals of $0.5 \mathrm{~mm}$ in thickness were used. First, we deposited a thin layer of photoresist onto the $+Z$ surface of the crystal, which was then lithographically patterned with the designed chirped lattices. The exposed area was brought into contact with liquid electrolyte, which consisted of a saturated solution of lithium chloride in deionized water. The unexposed area was separated from the liquid electrolyte by the photoresist. Then, a pulsed electric field of approximately $24 \mathrm{kV} \mathrm{mm}^{-1}$ was applied on two sides of the crystal to complete the domain reversion. We etched the sample in hydrofluoric acid for $10 \mathrm{~min}$ to make it possible to observe the poled pattern using a microscope.

\section{Measurement of the nonlinear conversion efficiency}

The SHG and THG conversion efficiencies of the CPPLN samples were measured using a tunable optical parametric oscillator pumped by a $1064 \mathrm{~nm}$ Nd:YAG laser. The average optical parametric oscillator output was approximately 5-20 $\mathrm{mW}$ at $850-1450 \mathrm{~nm}$, and the pulse width and repetition rate were $3.5 \mathrm{~ns}$ and $10 \mathrm{~Hz}$, respectively. The fundamental wave (FW) was weakly focused with a spot radius of approximately $200 \mu \mathrm{m}$ and was coupled into the polished end of the sample. The peak value of the FW pump power was at the modest level of $0.1 \mathrm{GW} \mathrm{cm}^{-2}$. Then suitable filters were used to separate the fundamental wave and the harmonics, and a power meter was used to record the pulse power, from which the conversion efficiency was calculated.

\section{RESULTS AND DISCUSSION}

The conversion efficiencies for SHG and THG were first evaluated theoretically by solving the standard nonlinear coupled-wave equations for the FW, the second-harmonic wave (SHW) and the thirdharmonic wave (THW). The detailed mathematical formulations are provided in the Supplementary Information, and a typical result for the nonlinear wave evolution including the FW, the SHW and the THW is illustrated in Supplementary Fig. S1. The calculation results for the three different chirp rates are presented in Figure 2. The phasecompensation scheme in our chirped QPM structure is explicitly noted in the inset for each conversion-efficiency peak. From Figure 2a for SHG, it is evident that our structure can achieve QPM at various orders for SHG, and as $D_{g}$ increases, the bandwidth widens but the conversion efficiency decreases. There are four wavelength regions around central wavelengths of $1.4,1.1,0.97$ and $0.89 \mu \mathrm{m}$, which correspond to the first-, second-, third- and fourth-order QPM processes, respectively. The bandwidths of the four orders for $D_{g}=0.6$ are $25,15,14$ and $12 \mathrm{~nm}$, respectively. For $D_{g}=1.2$, they are 48, 31,26 and $24 \mathrm{~nm}$, respectively, whereas for $D_{g}=2.4$, they increase to 97, 63, 51 and $46 \mathrm{~nm}$, respectively. The conversion efficiencies for the four orders for $D_{g}=0.6$ are approximately $70 \%, 20 \%, 7 \%$ and $8 \%$, respectively. For $D_{g}=1.2$, they are $60 \%, 10 \%, 4 \%$ and $4 \%$, respectively, whereas for $D_{g}=2.4$, they are $40 \%, 5 \%, 2 \%$ and $2 \%$, respectively.

The bandwidth broadening and the tradeoff between bandwidth and conversion efficiency can also be clearly observed in Figure 2b for THG. All these phenomena are closely related to the characteristics
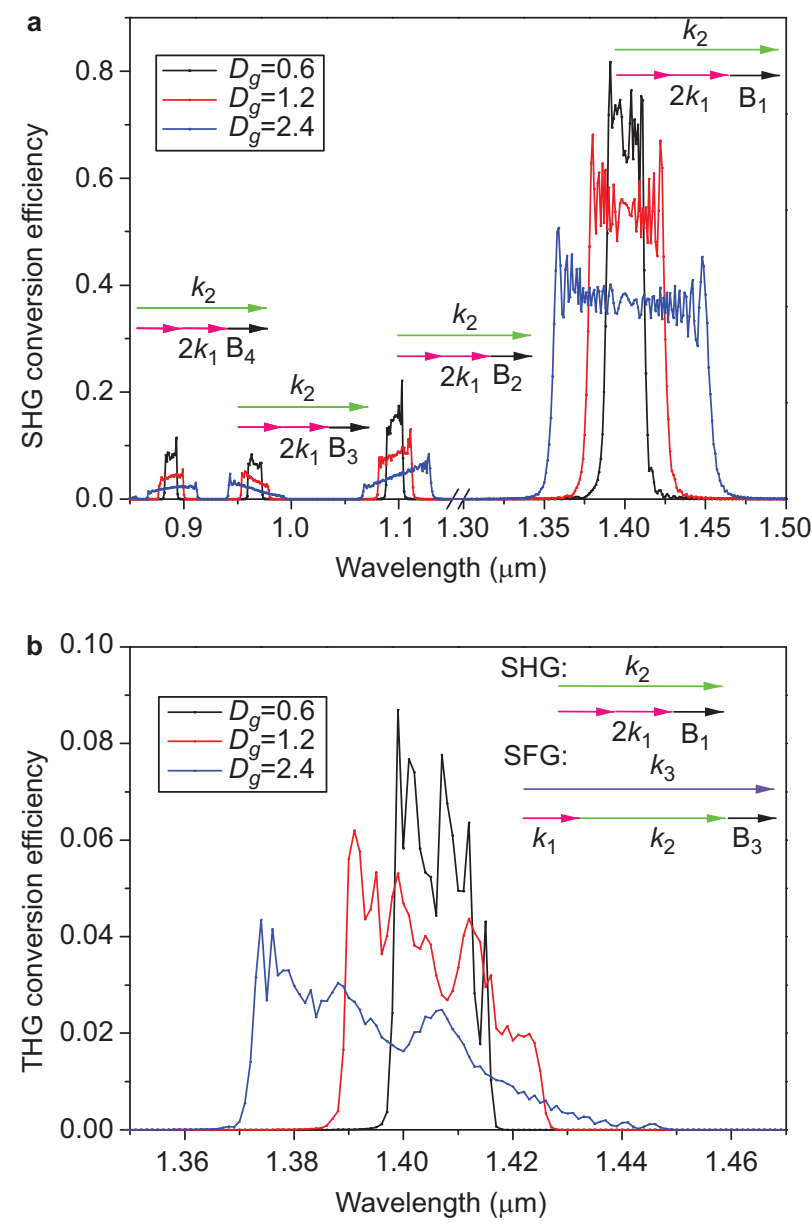

Figure 2 Evaluation of SHG and THG in CPPLN samples. Theoretical results for the conversion efficiency vs. the wavelength of incident FW light for (a) SHG and (b) THG under a pumping power of $0.05 \mathrm{GW} \mathrm{cm}^{-2}$ and for a sample length of $16 \mathrm{~mm}$. The inset figures explicitly indicate the QPM processes leading to SHG and THG. SHG can occur in four wavelength regions, which correspond to first- to fourth-order QPM, whereas THG can occur only in a single wavelength band, where the first-order QPM condition for SHG and the third-order QPM condition for SFG are simultaneously satisfied. CPPLN, chirped periodically poled lithium niobate; FW, fundamental wave; QPM, quasiphase matching; SHG, second-harmonic generation; THG, third-harmonic generation. 
of the RLV bands for the different chirp rates, as illustrated in Figure 1. The THG bandwidths are $18 \mathrm{~nm}$ for $D_{g}=0.6,36 \mathrm{~nm}$ for $D_{g}=1.2$ and $43 \mathrm{~nm}$ for $D_{g}=2.4$. The maximum bandwidth of $43 \mathrm{~nm}$ is achieved for $D_{g}=2.4$, and the maximum conversion efficiency of $8 \%$ is achieved for $D_{g}=0.6$. Although some tradeoff cannot be avoided, the CPPLN crystals still exhibit good overall performance in terms of both broad bandwidths and high conversion efficiencies. By comparison, a traditional PPLN structure can only provide discrete RLVs and thus cannot achieve frequency conversion over a broad spectrum of wavelengths.

We fabricated several CPPLN samples with different chirp rates using the electric poling technique at room temperature. Figure 3 presents a microscopic image of a typical CPPLN sample. Figure $3 \mathrm{a}$ illustrates that the negative domains are very uniform over the entire sample. Figure $3 \mathrm{~b}$ provides higher magnification images of two different parts of the sample, in which the reduction in the positive-domain width along the light-propagation direction can be clearly seen. The measured conversion efficiencies of SHG and THG are presented in Figures $4 a-4 c$ and $5 a-5 c$, respectively, over a broad range of wavelengths of the FW pumping light. Three CPPLN samples (samples 1, 2 and 3), with chirp rates $D_{g}$ of $0.6,1.2$ and 2.4, respectively, were evaluated and compared in great detail. It can be seen that for samples 1, 2 and 3, the SHG conversion efficiencies for first-order QPM are approximately $50 \%, 40 \%$ and $30 \%$, respectively, but the bandwidths are approximately 26, 46 and $98 \mathrm{~nm}$, respectively. The conversion efficiencies (bandwidths) of samples 1,2 and 3 for second-order QPM are approximately 25\% (16 nm), 20\% (24 nm) and 11\% $(58 \mathrm{~nm})$, respectively; for third-order QPM, they are approximately $14 \%(15 \mathrm{~nm}), 13 \%(29 \mathrm{~nm})$ and $8 \%(53 \mathrm{~nm})$, respectively. In the case of THG, the conversion efficiencies are approximately $4 \%, 3 \%$ and $2 \%$, and the bandwidths are approximately 21,42 and $74 \mathrm{~nm}$ for samples 1, 2 and 3, respectively. Clearly, when the chirp rate is increased, the bandwidth is broadened at the cost of a reduction in the conversion efficiency for both SHG and THG; these finding are in very good agreement with the theoretical results presented in Figure 2. The experimental conversion efficiency is lower than the theoretical value, and we believe that this is because the intensity of the incident light was not sufficiently high and because there was some energy loss during the measuring process. The discrepancy may also be related to a deviation of the nonlinear lattices in the real CPPLN samples from the ideal structure. ${ }^{25}$ Further numerical calculations indicate that the THG conversion efficiency significantly increases when the FW

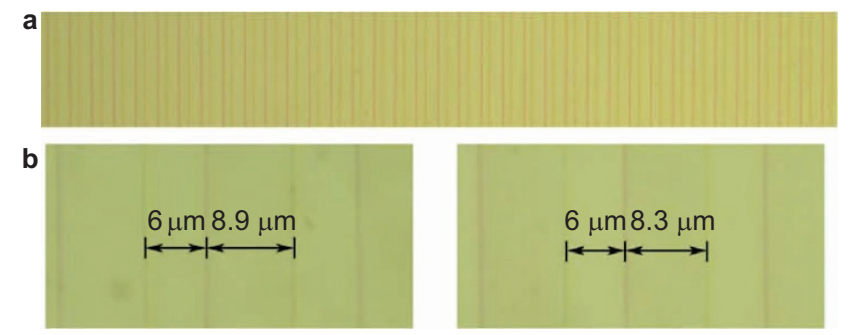

Figure 3 Fabrication of CPPLN samples. (a) Microscopic image of the etched sample surface of a typical CPPLN structure, illustrating the high uniformity of the nonlinear ferroelectric domains. (b) High-magnification images of two small segments from the left-hand and right-hand portions of the CPPLN sample, in which it can be seen that the negative domains are identical in size and the positive domains decrease in size from left to right, giving rise to the chirped poling period. CPPLN, chirped periodically poled lithium niobate.

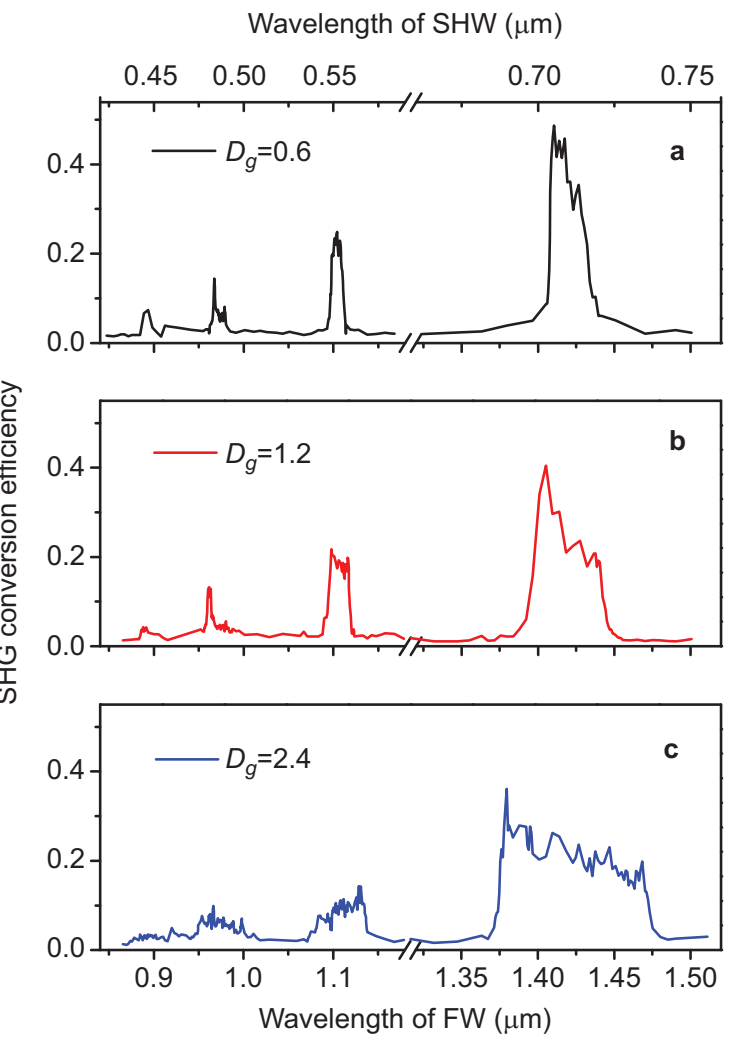

d

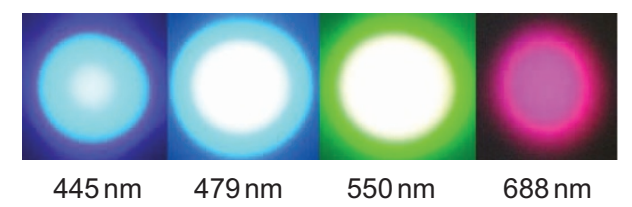

Figure 4 SHG in CPPLN samples. (a-c) Measured conversion efficiencies for SHG as a function of the pumping wavelength for three CPPLN samples with different chirp rates. (d) Photographs of several SHW beam spots emitted from the CPPLN samples exhibiting colors ranging from purple to red, which correspond to the four SHG bands. FW, fundamental wave; CPPLN, chirped periodically poled lithium niobate; SHG, second-harmonic generation; SHW, second-harmonic wave.

pumping power and the sample length are increased, as illustrated in Supplementary Fig. S2.

The output SHW and THW beams radiating from the CPPLN samples were recorded using cameras. When the incident wavelength was continuously tuned from 850 to $1480 \mathrm{~nm}$, SHG was constantly observed with a color that changed continuously from purple to red, and THG was observed with a continuous change from blue to green when the incident wavelength was tuned from 1360 to $1610 \mathrm{~nm}$. Photographs of SHW and THW beams are provided in Figures 4d and $5 \mathrm{~d}$, respectively, for several typical wavelengths. The incident FW light was a Gaussian beam, and all SHW and THW beams exhibited good modal profiles consistent with Gaussian beams. These results strongly indicate that our CPPLN samples have high ferroelectricdomain uniformity such that the scattering of FW, SHW and THW light caused by disorders, defects, roughness and non-uniformity in the sizes of the positive and negative ferroelectric domains in the CPPLN samples is negligible in the directions both parallel (namely, the $z$ axis) and perpendicular (namely, the $x$ and $y$ axes) to the lightpropagation direction. Note that our CPPLN samples exhibit four 


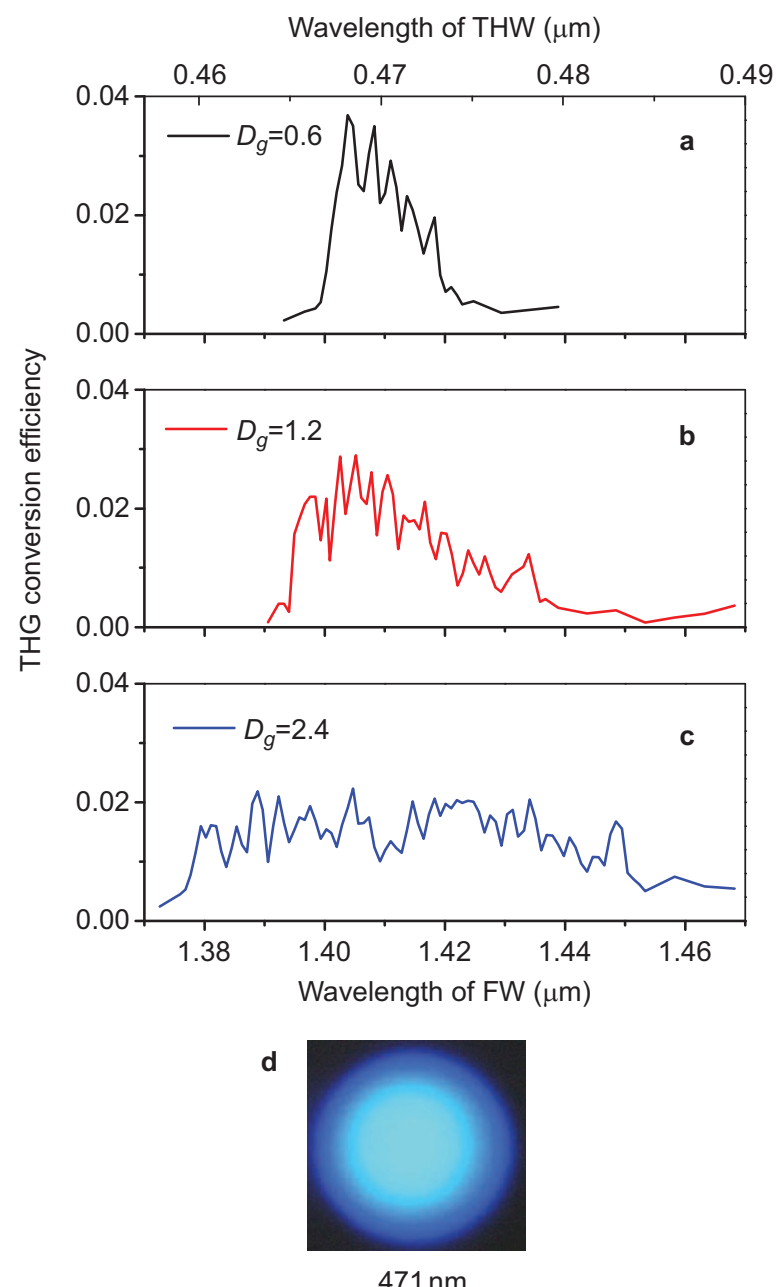

Figure 5 THG in CPPLN samples. (a-c) Measured conversion efficiencies for THG as a function of the pumping wavelength for three CPPLN samples with different chirp rates. (d) Photograph of a typical THW beam spot emitted from the CPPLN samples. CPPLN, chirped periodically poled lithium niobate; FW, fundamental wave; THG, third-harmonic generation; THW, third-harmonic wave.

SHG bands- $(442-449 \mathrm{~nm}),(481-492 \mathrm{~nm}),(547-555 \mathrm{~nm})$ and (703$718 \mathrm{~nm}$ - —which correspond to four color regions: purple $(380-450 \mathrm{~nm})$, blue (450-495 nm), green (495-570 nm) and red (620-750 nm). This means that a single CPPLN sample can simultaneously generate three primary colors (red, green and blue), and this functionality may have potential applications in laser displays.

Our CPPLN structure offers several RLV bands to support various second-order QPM nonlinear processes such as SHG and SFG, leading to the realization of higher-order processes such as THG. Although other structures, such as quasiperiodic lattices, ${ }^{7,11}$ can perform similar tasks, our chirped structures support a finite bandwidth for each RLV band, and this feature strongly facilitates THG because there is no need for stringent accuracy in ferroelectric-domain fabrication to ensure that the QPM conditions are satisfied. Clearly, this CPPLN scheme is also applicable to harmonic generation at even higher orders with a broad, tunable bandwidth and high frequency-conversion efficiency.

\section{CONCLUSIONS}

We have fabricated CPPLN crystals composed of negative domains of fixed-width and variable-width positive domains. Because the period is deliberately chirped, these structures provide several continuous RLV bands with large effective nonlinear susceptibility, thereby enabling simultaneous SHG and THG from a single sample. Both experimental observations and theoretical calculations indicate that these samples exhibit high performance in terms of a balance between high efficiency and broad bandwidth. The samples support not only the typical firstorder QPM process but also several higher-order QPM processes that are required for SHG and SFG, whose position and bandwidth can be controlled as desired. As a result, a single CPPLN crystal can generate three primary colors with a broad bandwidth. In addition, a CPPLN sample can support QPM for both SHG and SFG in the same wavelength band, leading to simultaneous broadband SHG and THG from a single crystal. The maximum SHG bandwidth is $98 \mathrm{~nm}$ with a conversion efficiency of $30 \%$, and the maximum THG bandwidth is $74 \mathrm{~nm}$ with a conversion efficiency of $2 \%$. These results strongly indicate that these CPPLN structures are good candidates for use as short-wavelength laser sources in applications related to large-screen laser display technology and photoelectron spectrometers.

\section{ACKNOWLEDGMENTS}

This work was supported by the 973 Program of China (No. 2011 CB922002 and No. 2013CB632704) and the Knowledge Innovation Program of the Chinese Academy of Sciences (No. Y1 V2013 L11).

1 Franken PA, Hill AE, Peters CW, Weinreich G. Generation of optical harmonics. Phys Rev Let 1961; 7: 118-120.

2 Armstrong JA, Bloembergen N, Ducuing J, Pershan PS. Interactions between light waves in a nonlinear dielectric. Phys Rev 1962; 127: 1918-1939.

3 Fejer MM, Magel GA, Jundt DH, Byer RL. Quasi-phase-matched second harmonic generation: tuning and tolerances. IEEE J Quantum Electron 1992; 28: 2631-2654.

4 Berger V. Nonlinear photonic crystals. Phys Rev Lett 1998; 81: 4136-4139.

5 Broderick NG, Ross GW, Offerhaus HL, Richardson DJ, Hanna DC. Hexagonally poled lithium niobate: a two-dimensional nonlinear photonic crystal. Phys Rev Lett 2000; 84: 4345-4348

6 Piskarskas A, Smilgevicius V, Stabinis A, Jarutis V, Pasiskevicius V et al. Noncollinear second-harmonic generation in periodically poled $\mathrm{KTiOPO}_{4}$ excited by the Bessel beam. Opt Lett 1999; 24: 1053-1055.

$7 \quad$ Ma BQ, Wang T, Sheng Y, Ni PG, Wang YQ et al. Quasiphase matched harmonic generation in a two-dimensional octagonal photonic superlattice. Appl Phys Lett 2005; 87: 251103.

8 Ren ML, Ma DL, Li ZY. Experimental demonstration of super quasi-phase matching in nonlinear photonic crystal. Opt Lett 2011; 36: 3696-3698.

9 Tehranchi A, Kashyap R. Design of novel unapodized and apodized step-chirped quasi-phase matched gratings for broadband frequency converters based on second-harmonic generation. J Lightwave Tech 2008; 26: 343-349.

10 Kintaka K, Fujimura M, Suhara T, Nishihara H. Third harmonic generation of Nd:YAG laser light in periodically poled $\mathrm{LiNbO}_{3}$ waveguide. Electron Lett 1997; 33: 14591461.

11 Zhu SN, Zhu YY, Ming NB. Quasi-phase-matched third-harmonic generation in a quasi-periodic optical superlattice. Science 1997; 278: 843-846.

$12 \mathrm{Mu} X \mathrm{X}$, Ding YJ. Efficient third-harmonic generation in partly periodically poled $\mathrm{KTiOPO}_{4}$ crystal. Opt Lett 2001; 26: 623-625.

13 Ma BQ, Wang T, Ni PG, Cheng BY, Zhang DZ. High-order quasi-phase-matching harmonic generation in two-dimensional orthorhombic lattice. Europphys Lett 2004; 68: 804-810.

14 Saltiel SM, Sukhorukov AA, Kivshar YS. Multistep parametric processes in nonlinear optics. Progress in Optics 2005; 47: 1-73.

15 Tehranchi A, Kashyap R. Engineered gratings for flat broadening of second-harmonic phase-matching bandwidth in MgO-doped lithium niobate waveguides. Opt Express 2008; 16: 18970-18975.

16 Mizuuchi K, Yamamoto K, Kato M, Sato H. Broadening of the phase-matching bandwidth in quasi-phase-matched second-harmonic generation. IEEE J Quantum Electron 1994; 30: 1596-1604.

17 Akhamanov SA, Vyslouch VA, Chirkin AS. Optics of Femtosecond Laser Pulses. New York: American Institute of Physics; 1992.

18 Arbore MA, Macro O, Fejer MM. Pulse compression during second-harmonic generation in aperiodic quasi-phase-matching gratings. Opt Lett 1997; 22: 865867.

19 Arbore MA, Galvanauskas A, Harter D, Chou MH, Fejer MM. Engineerable compression of ultrashort pulses by use of second-harmonic generation in chirped-period-poled lithium niobate. Opt Lett 1997; 22: 1341-1343. 
20 Markowicz PP, Tiryaki H, Pudavar H, Prasad PN, Lepeshkin NN et al. Dramatic enhancement of third-harmonic generation in three-dimensional photonic crystals. Phys Rev Lett 2004; 92: 083903.

21 Artigas D, Reid DT. Efficient femtosecond optical parametric oscillators based on aperiodically poled nonlinear crystals. Opt Lett 2002; 27: 851-853.

22 Yusupov DB, Chirkin AS. Frequency doubling of phase-modulated femtosecond laser pulses in periodically poled and chirped nonlinear crystals. Phys Wave Phenom 2007; 15: 263-271.

23 Yusupov DB. Degenerate parametric frequency conversion of phase-modulated femtosecond laser pulses in crystals with a regular and chirped domain structure. Laser Phys 2008; 18: 43-51.

24 Ren ML, Li ZY. An effective susceptibility model for exact solution of second harmonic generation in general quasi-phase-matched structures. EPL 2011; 94: 44003.
25 Shutov IV, Chirkin AS. Simulation of the random violation of the quasi-phasematching condition in optical parametric process. Quantum Electron 2009; 39: 691-696.

(i) (8) This work is licensed under a Creative Commons Attribution-

NonCommercial-NoDerivs 3.0 Unported License. The images or other third party material in this article are included in the article's Creative Commons license, unless indicated otherwise in the credit line; if the material is not included under the Creative

Commons license, users will need to obtain permission from the license holder to reproduce the material. To view a copy of this license, visit http://creativecommons.org/licenses/ by-nc-nd/3.0/

Supplementary information for this article can be found on the Light: Science \& Applications' website (http://www.nature.com/lsa/). 